\title{
Escala de Perceção dos Alunos sobre o seu Envolvimento Comportamental Escolar: Construção e Validação ${ }^{1}$
}

\author{
Carolina Carvalho ${ }^{2}$ \\ Joseph Conboy \\ João Santos \\ Jesuína Fonseca \\ Universidade de Lisboa \\ David Tavares \\ Escola Superior de Tecnologia da Saúde de Lisboa-Instituto Politécnico de Lisboa; \\ CIES-IUL Instituto Universitário de Lisboa \\ Dulce Martins \\ Instituto Universitário de Lisboa (ISCTE-IUL), \\ Centro de Investigação e Intervenção Social; UIDEF, \\ Instituto de Educação, Universidade de Lisboa, Portugal \\ Maria Helena Salema \\ Universidade de Lisboa \\ Edite Fiúza \\ Universidade Lusófona de Humanidades e Tecnologias \\ Ana Paula Gama \\ Instituto Superior Dom Afonso III
}

\begin{abstract}
RESUMO - Este estudo descreve a construção e validação de uma escala que avalia a perceção dos alunos sobre o seu envolvimento comportamental escolar. Os questionários foram aplicados a 1089 alunos do $6^{\circ}, 7^{\circ}, 9^{\circ}$ e $10^{\circ}$ anos de escolaridade (idade mediana $=13$ ) em Portugal Continental, a partir dos quais foram extraídas duas subamostras aleatórias. Uma subamostra foi submetida a análise fatorial exploratória e a segunda, a uma análise fatorial confirmatória. Emergiram duas dimensões: envolvimento no trabalho acadêmico e participação na aula. A primeira escala revelou uma consistência interna aceitável, revelando-se útil e adequada para avaliar as percepções dos alunos sobre o seu envolvimento comportamental escolar. A segunda dimensão necessita de maior desenvolvimento em futuras investigações.
\end{abstract}

Palavras-chave: estudantes, características dos estudantes, rendimento escolar, modelação de equações estruturais

\section{Scale of Student Perceptions Regarding their Behavioral Engagement in School: Construction and Validation}

\begin{abstract}
This study presents the construction and validation of a scale evaluating student perceptions regarding their behavioral engagement in school. The questionnaire was administered to 1089 students attending the 6th, 7th, 9th and 10th grades (median age $=13$ years) in Continental Portugal, from which two samples were randomly selected. One of the samples was submitted to exploratory factor analysis; the second sample was submitted to confirmatory factor analysis. Two dimensions emerged: involvement in academic work and class participation. The first scale presented acceptable internal consistency, and is useful and appropriate to evaluate students' perceptions regarding their behavioral engagement in school. The second dimension requires further research and development.
\end{abstract}

Keywords: students, student characteristics, academic achievement, structural equation modeling

O envolvimento do aluno nas atividades escolares pode representar um fator decisivo no sucesso e na prevenção do abandono escolar (Kinderman, 2007), sendo por isso considerado um indicador poderoso para o seu projeto de vida futuro. Uma revisão da literatura revela a existência da associação entre o envolvimento escolar e a permanência na escola bem como o sucesso acadêmico dos alunos (Fredricks, Blumenfeld, \& Paris, 2004). Estudos mais

1 Esta investigação teve o apoio da FCT (contrato PTDC/CPE-PEC/121238/2010)

2 Endereço para correspondência: Instituto de Educação, Universidade de Lisboa, Alameda da Universidade, 1649-013, Lisboa, Portugal. E-mail: cfcarvalho@ie.ul.pt recentes corroboram essas conclusões e apresentam evidência de uma associação entre envolvimento escolar e resultados de avaliações acadêmicas (Dotterer \& Lowe, 2011; Finn \& Zimmer, 2012), comportamentos desviantes ou delinquentes (Hirschfield \& Gasper, 2011; Li, et al., 2011; Simons-Morton \& Chen, 2009) e risco de abandono escolar (Archambault, Janosz, Morizot, \& Pagani, 2009). O envolvimento dos alunos aumenta a probabilidade de despertar curiosidade, otimismo e gosto pela aprendizagem, traços que normalmente surgem acompanhados por uma preferência pela ação, pelo esforço e pela persistência (Skinner \& Belmont, 1993).

Uma operacionalização do envolvimento inclui normalmente a mensuração de seus componentes 
comportamentais e afetivos (e.g. Handelsman, Briggs, Sullivan, \& Towler, 2005; Skinner, Kinderman, \& Furrer, 2009). Skinner et al. (2009) sugerem a seguinte definição:

Em termos gerais, o envolvimento refere-se à qualidade da relação do aluno com o empreendimento escolar, portanto com as pessoas, actividades, objetivos, valores, e local que o compõem (...) o envolvimento representa uma influência próxima potencialmente modeladora dos níveis de retenção, sucesso e resiliência dos alunos. Apesar de um emergente consenso quanto ao quadro geral, no entanto, o trabalho desenvolvido sobre o envolvimento, porque reflecte múltiplas perspectivas, trouxe consigo uma profusão de definições conceptuais e operacionais. Na raiz de diversas conceptualizações encontrase um construto que se refere à qualidade da participação dos alunos nas actividades de aprendizagem em sala de aula, desde as interacções mais enérgicas, entusiastas, focadas, e emocionalmente positivas em relação às tarefas académicas, até aquelas caracterizadas por um afastamento apático. (p. 494)

O envolvimento escolar do aluno revela-se um construto que se reveste de um interesse particular para os educadores, uma vez que se trata de um fator que - ao contrário de variáveis preditivas como o sexo, etnia ou classe social se apresenta como maleável e, por isso, parcialmente sob o controle dos professores e das escolas. No âmbito dos contextos de participação, o envolvimento escolar do aluno surge como uma variável fortemente associada com o sucesso escolar e o abandono escolar (Fredricks et al., 2004).

A literatura foca-se sobretudo nas dimensões comportamentais, afetivas e cognitivas. Contudo, recentemente alguns autores vêm sugerir a existência de uma quarta dimensão, agenciativa, conceitualizada como o contributo construtivo dos alunos à instrução recebida (Reeve \& Tseng, 2011; Veiga, 2013).

Em específico, e porque será a componente mais focada no presente estudo, a componente comportamental do envolvimento escolar traduz-se nas ações do aluno em contexto acadêmico, nas suas práticas e atividades escolares (Veiga, Galvão, Festas, \& Taveira, 2012), como a realização dos trabalhos de casa (Finn \& Rock, 1997), a frequência e atenção nas aulas (Johnson, Crosnoe, \& Elder, 2001), o empenho nas tarefas escolares, a obtenção de boas notas (Jordan \& Nettles, 2000), a participação em atividades extracurriculares (Finn, 1993), o respeito pelas regras da escola e a ausência de comportamento disruptivo (Fredricks et al., 2004).

Em um estudo realizado por Skinner et al. (2009), verificou-se que os alunos reportaram um maior envolvimento escolar comparativamente ao que foi atribuído pelos professores, e que os professores subestimaram os níveis de desapego afetivo dos alunos, em comparação aos relevados pelos próprios alunos. Esses resultados sugerem diferenças nas percepções de alunos e professores em relação ao que ocorre nas aulas. Sugerem que pode ocorrer da parte dos professores uma sistemática desvalorização do esforço despendido pelos alunos (ou, inversamente, que os alunos poderão sobrevalorizar os seus esforços).

Newmann (1992) identificou vários fatores no contexto escolar que podem influenciar o envolvimento dos alunos. Alguns desses fatores salientavam variáveis organizacionais e incluíam os objetivos escolares, a justiça implícita nas práticas, o apoio individual, a existência de um ambiente preocupado e seguro, o sentimento de pertença, uma clara ligação com o mundo real e o "divertimento". No que se refere à sala de aula, o mesmo autor sugeriu que o envolvimento escolar pode ser incentivado por atividades que os alunos sintam como cabíveis, permitam-lhes uma sensação de controle e possam ser agradáveis, além de possibilitarem a colaboração entre pares e a utilização de diferentes capacidades.

O envolvimento surge também associado com a identificação dos alunos com a escola.

Na prática, o envolvimento traduz-se quer naquilo que fazemos, quer nas relações que estabelecemos com os outros, permitindo-nos perceber qual a nossa competência para nos relacionarmos e o que somos ou não capazes de fazer, contribuindo, assim, para a nossa perceção de quem somos, desenvolvendo e moldando a nossa identidade (Wenger, 2007).

A fim de estudar as percepções dos alunos sobre o seu envolvimento escolar, em específico em seu componente comportamental, bem como uma eventual associação com o contexto escolar e com fatores como a identificação escolar e as trajetórias escolares, é necessário desenvolver uma medida objetiva de como os alunos percebem o seu envolvimento comportamental escolar. O presente estudo pretende descrever o processo de construção e validação de uma escala que visa a avaliar essas percepções.

\section{Método}

\section{Amostragem}

A população alvo do presente estudo consiste de alunos do ensino básico e secundário que frequentam anos de transição entre ciclos de estudo ${ }^{1}$. Assim, pretendendo-se uma amostra ampla e abrangente, que considerasse a diversidade inerente ao universo dos alunos que frequentam os $6^{\circ}, 7^{\circ}, 9^{\circ}$ e $10^{\circ}$ anos, constituiu-se a amostra através de um processo de estratificação multiestágio, em Portugal Continental.

Em uma primeira fase, procedeu-se a um levantamento da informação relativa ao número de alunos matriculados nos ensinos básico e secundário do ensino regular de acordo com o nível de ensino, o ciclo de estudo e o ano de escolaridade, por Unidades Territoriais para Fins Estatísticos (NUTS II dados referentes ao ano letivo de 2010/2011). Em seguida, procedeu-se ao levantamento de informação relativa a todos os estabelecimentos de ensino regular (públicos e privados), constituindo-se uma listagem das escolas, segundo o nível de ensino, por NUTS II, distrito e concelho (esses dados foram

1 O Sistema de Ensino Português está dividido em cinco ciclos de estudo; ensino pré-escolar (crianças entre os 3 e os 6 anos), ensino básico (dividido em três ciclos. O primeiro compreende quatro anos, o segundo, dois e o terceiro, três anos letivos), ensino secundário (com três anos orientado para alunos que visam prosseguir estudos), ensino secundário não-superior (possibilita formação especializada em diferentes áreas tecnológicas, permitindo a inserção no mundo do trabalho ou no ensino superior), ensino superior. 
obtidos através do Roteiro das Escolas, situado no Portal da Educação do Ministério da Educação de Portugal).

Em uma segunda fase, a partir do total dos alunos matriculados nos $6^{\circ}, 7^{\circ}, 9^{\circ}$ e $10^{\circ}$ anos, por NUTS II, procedeuse ao cálculo da percentagem de alunos, por NUTS II, em cada um dos anos de escolaridade. Através das percentagens obtidas, foi então calculado, em proporção, o número de alunos a serem selecionados para participar no estudo, por NUTS II e ano de escolaridade. Agregou-se a essa informação o número total de escolas que ofereciam, respectivamente, os $6^{\circ}, 7^{\circ}, 9^{\circ}$ e $10^{\circ}$ anos, por concelho e NUTS II.

$\mathrm{O}$ passo seguinte consistiu na seleção aleatória de $50 \%$ dos concelhos, agregados por NUTS II, para cada ano de escolaridade. Compilou-se então uma listagem das escolas situadas nos concelhos selecionados, a partir da qual se selecionaram 5\% das escolas. Esse passo permitiu obter o número e lista de escolas, por NUTS II, por ano de escolaridade, onde foi aplicado o inquérito por questionário. Finalmente procedeu-se à definição do número de turmas necessárias por cada escola (esta estimativa teve por base o pressuposto, conservador, de que, por cada turma, seriam recolhidos 15 questionários e tendo em consideração o número mínimo requerido de alunos necessários para a amostra por NUTS II e ano de escolaridade).

\section{Participantes}

A amostra final foi constituída por 1089 alunos, provenientes do ensino regular, especificamente, do $6^{\circ}$ ano $(25,7 \%), 7^{\circ}$ ano $(31,7 \%), 9^{\circ}$ ano $(26,6 \%)$ e $10^{\circ}$ ano $(16 \%)$, distribuídos por 45 estabelecimentos de ensino público do território de Portugal Continental. As idades variam dos 10 aos 25 anos $(M=13,4$ e $D P=1,7 ; M d n=13,0)$, sendo que $41,4 \%$ têm idades compreendidas entre os 10 e os 12 anos, 46,8\% têm idades compreendidas entre os 13 e os 15 anos e $11,8 \%$ têm idades superiores a 15 anos. Essa amostra é constituída por alunos, na sua grande maioria $(95,9 \%)$, de nacionalidade portuguesa, sendo que $52 \%$ são do sexo feminino.

\section{Instrumento}

O questionário utilizado neste estudo foi desenvolvido por uma equipe multidisciplinar, que incluiu psicólogos (de educação e clínicos), professores (de educação em ciências) e sociólogos. A construção da escala de envolvimento comportamental seguiu várias etapas. A identificação e seleção de itens foram baseadas em investigação prévia (e.g. Finn \& Rock, Fredricks et al., 2004; 1997; Johnson et al., 2001; Veiga et al., 2012). Os itens resultantes foram apresentados como afirmações às quais os alunos se posicionavam em uma escala de quatro pontos, ancorada semanticamente nos extremos $($ Sempre $=3$; Nunca $=0)$.

Os cuidados tidos com a validade de conteúdo da escala (Haynes, Richard, \& Kubany, 1995) corresponderam a preocupações relacionadas com a elaboração e ordenação dos itens, bem como à forma das respostas. Quanto à elaboração dos itens, houve um cuidado particular no sentido de se evitarem: (a) utilização de termos que influenciassem e/ou induzissem as respostas; (b) utilização de termos ambíguos cujo significado não fosse percebido da mesma forma por todos os respondentes; (c) formulação de questões duplas; d) utilização de linguagem técnica dificilmente descodificável pelos respondentes; (e) desequilíbrio nas hipóteses de resposta. Relativamente à ordenação dos itens, a principal preocupação residiu no efeito de halo, ordenando-se os itens de forma a tentar garantir que a sua sequência não influenciasse as respostas (Tavares, 2007).

Foi então efetuado um teste piloto junto a alunos dos $6^{\circ} \mathrm{e}$ $7^{\circ}$ anos do ensino básico e $10^{\circ}$ ano do ensino secundário, na Área Metropolitana de Lisboa. A realização desse teste piloto teve como finalidade a obtenção de indicações acerca da forma como as perguntas são compreendidas ainda em uma fase reversível, anterior ao seu lançamento, e compreende também a aferição de fatores relativos, por exemplo, à ordem das perguntas, às recusas ou hesitações nas respostas a determinadas perguntas ou à reação geral ao questionário (Tavares, 2007).

A escala integra 10 itens focalizando o envolvimento comportamental escolar. Nesta os alunos deveriam responder em que medida afirmações como "Faço os trabalhos de casa" ou "Faço perguntas quando não compreendo a matéria" descrevem a sua realidade enquanto alunos. A fim de contextualizar as respostas, cada item foi apresentado com a instrução "Pensa numa disciplina de que GOSTAS e responde sempre a pensar na mesma". O questionário também incluiu uma seção sociodemográfica e uma seção sobre as trajetórias escolares do aluno.

No início da primeira página de cada questionário, informou-se os participantes por escrito do objetivo do estudo. Solicitou-se a sua participação voluntária e garantiu-se a confidencialidade das suas respostas. Nenhuma informação pessoal, potencialmente identificadora, foi recolhida.

\section{Procedimentos}

O levantamento de dados foi autorizado pelo Departamento de Monitorização de Inquéritos em Meio Escolar da Direção Geral de Inovação e Desenvolvimento Curricular. Após contato telefónico, enviou-se para as escolas, via correio, toda a documentação (questionários, pedidos de autorização a serem entregues aos encarregados de educação, instruções para os professores lerem às suas turmas, instruções para as direções das escolas e envelope com franquia para devolução dos questionários).

Com o objetivo de efetuar análises exploratórias e confirmatórias, dividiu-se a base de dados, aleatoriamente, ao meio. Nos dois subgrupos resultantes, foram removidos os casos com dados omissos. Os itens da escala de Perceção sobre o Envolvimento Comportamental Escolar (PECE) foram submetidos em primeiro lugar a uma Análise Fatorial Exploratória (AFE), que designamos por Estudo 1, seguindome uma Análise Fatorial Confirmatória (AFC) no Estudo 2. A realização da $\mathrm{AFC}$ visa testar o modelo estrutural proposto para descrever a dimensionalidade da escala Para realização 
das análises estatísticas, recorreu-se ao software SPSS 22.0 e AMOS 22.0.

\section{Estudo 1}

\section{Método}

Participantes. A amostra foi constituída por 354 alunos provenientes do ensino regular, especificamente, do $6^{\circ}$ ano (24\%), $7^{\circ}$ ano $(33,1 \%), 9^{\circ}$ ano $(26,6 \%)$ e $10^{\circ}$ ano $(16,4 \%)$. As idades variam dos 11 aos 25 anos $(M=13,4$ e $D P=1,7$;
Seis itens ficaram agregados ao primeiro fator, agregandose os três itens restantes ao segundo fator. Para o primeiro fator, designado como envolvimento no trabalho acadêmico (ETA), obteve-se um $\alpha=0,73$. Para o segundo fator, designado como participação na aula (PA), obteve-se $\alpha=$ 0,62 . Finalmente, verificou-se uma correlação moderada e estatisticamente significativa entre ETA e PA $(r=0,45 ; p<$ 0,001).

Tabela 1. Pesos Fatoriais dos Itens nos Dois Fatores (Rotação Varimax)

\begin{tabular}{lll}
\hline Item & $E T A$ & $P A$ \\
\hline 1. Estudo a matéria dada nas aulas & 0,81 & $-0,01$ \\
2. Presto atenção nas aulas & 0,71 & 0,20 \\
3. Faço os trabalhos de casa & 0,65 & 0,06 \\
4. Esforço-me por compreender a matéria, mesmo quando é difícil & 0,61 & 0,29 \\
5. Escrevo apontamentos nas aulas & 0,51 & 0,29 \\
6. Vou às aulas por vontade própria & 0,49 & 0,29 \\
7. Participo ativamente em discussões de grupoa & 0,08 & 0,78 \\
8. Levanto o braço para responder a uma pergunta & 0,16 & 0,70 \\
9. Faço perguntas quando não compreendo a matéria & 0,23 & 0,69 \\
\hline
\end{tabular}

$M d n=13$ ), sendo que 39,9\% têm idades compreendidas entre os 10 e os 12 anos, $49 \%$ têm idades compreendidas entre os 13 e os 15 anos e $11,1 \%$ têm idades superiores a 15 anos. Essa amostra é constituída por alunos, na sua grande maioria $(97,4 \%)$, de nacionalidade portuguesa, sendo que 55\% são do sexo feminino.

Procedimento. Procedeu-se, em primeiro lugar, à análise das correlações item-total da escala, para efeitos de sua depuração. Os itens foram então submetidos a uma AFE com extração de fatores pela técnica dos componentes principais e rotação ortogonal (varimax), obrigando os itens a uma associação exclusiva a apenas um fator. Como critério de exclusão de itens, definiu-se o peso fatorial de 0,30 como o mínimo para a aceitação de cada item. Também se recorreu à análise da consistência interna (alpha de Cronbach) das subescalas que operacionalizavam os fatores, excluindo-se os itens cuja correlação com o total da sua subescala fosse inferior a 0,20 .

\section{Resultados}

Após análise da correlação item-total da escala, optou-se pela exclusão de um item que apresentava uma fraca correlação $(r \leq 0,20)$. Os nove itens restantes foram submetidos a uma AFE, com rotação varimax e usando o critério de término de Kaiser, que resultou na extração de dois fatores, que explicavam $49 \%$ da variância total dos resultados. Na Tabela 1, apresentam-se os loadings dos itens nos dois fatores extraídos.

\section{Estudo 2}

\section{Método}

Participantes. A amostra foi constituída por 388 alunos, provenientes do ensino regular, especificamente, do $6^{\circ}$ ano $(22,9 \%), 7^{\circ}$ ano $(27,6 \%), 9^{\circ}$ ano $(28,1 \%)$ e $10^{\circ}$ ano $(21,4 \%)$. As idades variam dos 11 aos 17 anos $(M=13,5$ e $D P=1,6$; $M d n=14)$, sendo que $38 \%$ têm idades compreendidas entre os 10 e os 12 anos, $49 \%$ têm idades compreendidas entre os 13 e os 15 anos e 13\% têm idades superiores a 15 anos. Essa amostra é constituída por alunos, na sua grande maioria, de nacionalidade portuguesa $(96,1 \%)$, sendo que $55 \%$ são do sexo feminino.

Procedimento. Nesta análise, assumiu-se então um modelo hipotético bidimensional constituído por duas variáveis latentes (ETA e PA) e respectivas variáveis observadas/itens. Pretendeu-se assim averiguar a adequabilidade do modelo teórico aos dados empíricos.

De forma a testar a identidade do modelo teórico, recorreu-se à estatística do Qui-quadrado $\left(\chi^{2}\right)$ e aos índices de adequabilidade do ajustamento CFI, RMSEA e PNFI. O $\chi^{2}$ permite ter uma ideia ampla sobre o ajuste do modelo, avaliando a magnitude da discrepância entre a matriz de covariância dos dados observados na amostra e a matriz de covariância implícita do modelo proposto. A hipótese nula do teste é de que o modelo se ajusta perfeitamente à população. Uma vez que se trata de um teste de significância estatística, é bastante sensível ao tamanho da amostra, sendo comum a 
rejeição do modelo quando é utilizada uma grande amostra como acontece no presente estudo.

Para contornar as limitações do $\chi^{2}$, também se recorreu à interpretação da estatística $\chi^{2} / d f$ e aos índices de adequabilidade do ajustamento CFI, RMSEA e PNFI. A estatística $\chi^{2} / d f$ (relative/normed chi-square; Wheaton, Muthen, Alwuin, \& Summers, 1977) permite contornar as limitações do $\chi^{2}$, revelando-se menos sensível ao tamanho da amostra. $\mathrm{O}$ valor de referência dessa estatística deve ser igual ou inferior a 2,0 (Tabachnick \& Fidell, 2007). O índice de aderência CFI (comparative fit índex; Bentler, 1990), tal como o NFI (normed-fit index; Bentler \& Bonnet, 1980), compara o modelo hipotético com o modelo independente (que considera um cenário onde não há nenhuma correlação entre qualquer par das variáveis observadas). A utilização do CFI em detrimento do NFI justifica-se pelo fato de o primeiro ser um dos índices menos sensíveis ao tamanho da amostra, sendo que valores superiores a 0,90 revelam um bom ajuste do modelo (Hu \& Bentler, 1999). Reconhecido como um dos critérios mais informativos sobre modelação de equações em estruturas de covariância, o RMSEA (root mean square of approximation; Steiger \& Lind citados por Steiger, 1990) tem em consideração o erro de aproximação na população. O RMSEA favorece a parcimónia, ou seja, opta normalmente pelo modelo com menor número de parâmetros, sendo que valores inferiores a 0,6 indicam um bom ajuste do modelo (Hu \& Bentler, 1999). Uma outra característica relevante do RMSEA diz respeito à estimação de intervalos de confiança (IC) calculados para o valor do índice, cujo limite inferior será próximo de 0 e o limite superior deverá ser menor que 0,08 (Hooper, Coughlan \& Mullen, 2008; Pilati \& Laros, 2007). Finalmente, o PNFI (parsimonious normed-fit índex; Mulaik et al., 1989) fornece informações sobre o ajuste do modelo por meio da relação entre o número de parâmetros estimados e a sua qualidade de aproximação na população. Trata-se de um índice que penaliza a complexidade do modelo, o que resulta em valores consideravelmente mais baixos em comparação a outros índices de ajuste do modelo. Mulaik et al. (1989) referem a possibilidade de obtenção de valores que rondam 0,50 , no entanto, uma vez que a literatura não refere o valor limite de PNFI que corresponda a um bom ajuste do modelo, torna-se mais difícil a sua interpretação e, como tal, este convém ser reportado em conjunto com outros índices de ajuste (Hooper et al., 2008).

Como base empírica para a melhoria de ajuste dos modelos teóricos a partir da sua reespecificação, quando necessário, foi considerada a inspeção dos índices de modificação, estratégia que permite excluir os itens cujo erro de mensuração correlaciona com o de outros itens, considerando o número de inter-relações e a sua contribuição para o decréscimo do $\chi^{2}$ (Diniz \& Almeida, 2005). Contudo, os índices de modificação não devem, por si só, guiar o processo de reespecificação de um modelo, devendo basearse em conhecimento teórico à priori (Hooper et al., 2008), pelo que, na sua ausência, optar-se-á por não os seguir. Também foi considerada a relação entre a variância dos itens associada à variância do respectivo factor $\left(R^{2}\right)$ e a variância dos itens que surge associada ao seu erro. Assim, sempre que o $R^{2}$ obtido para um dado item se revelou muito baixo $(\leq 0,15)$, este foi eliminado por ser pouco preciso. Para o estabelecimento da unidade de mensuração dos fatores de primeira ordem do modelo, o coeficiente de regressão nãoestandardizado de um dos seus itens foi igualizado a um $(1,00)$, passando a escala dos fatores a ser a mesma dos itens.

Finalmente, procedeu-se à análise da consistência interna (alpha de Cronbach) das subescalas (Cortina, 1993; Sijtsma, 2009). Para efeitos de investigação, os valores de 0,60 ou 0,70 para cima são considerados adequados (Clark \& Watson, 1995).

\section{Resultados}

Apesar do valor do $\chi^{2}$ ser significativo $(p<0,05)$, o modelo bidimensional hierárquico revelou-se um modelo ajustado, de acordo com as estatísticas $\chi^{2} / d f(1,76<2,0)$ e $C F I>0,95$, e com a probabilidade do $R M S E A<0,05>0,05$ (IC de $90 \%=0,022 ; 0,065)$.

A inspeção dos índices de modificação do modelo não denunciou a existência de intercorrelações de erros suficientemente fortes que justificassem o estabelecimento dessa relação no modelo. A análise da matriz dos resíduos estandardizados também não identificou nenhum item que estivesse a contribuir fortemente para o desajuste do modelo.

Com exceção do item $5(\beta=0,40)$, todos os outros itens registaram um peso fatorial de elevada magnitude $(\beta>0,50)$. O item 4 revelou ser o que melhor representava a ETA $\left(R^{2}\right.$ $=0,53)$, sendo o item 10 o que melhor representava a PA $\left(R^{2}=0,38\right)$. Relativamente à estrutura latente do modelo, verificamos que a PA era o fator que melhor representava a Perceção dos Alunos sobre o Envolvimento Comportamental Escolar $\left(R^{2}=0,85\right)$, a que se seguia a subescala ETA $\left(R^{2}\right.$ $=0,64)$.

A subescala que operacionaliza o ETA apresentou uma boa consistência interna $(\alpha=0,76)$. Verificou-se, no entanto, que a subescala que operacionaliza a PA apresentou uma fraca consistência interna $(\alpha=0,59)$.

Assim, a solução bidimensional revelou-se ajustada. No entanto, dadas as fragilidades da subescala PA (nomeadamente a baixa consistência interna), decidiuse efetuar ainda uma segunda análise exploratória com o objetivo de apresentar uma medida alternativa de envolvimento comportamental escolar, que poderá ser útil enquanto futuras investigações não desenvolvam melhor a solução bidimensional. Foi nesse sentido, exploratório, que testamos a alternativa unidimensional recorrendo à técnica de modelação de equações estruturais. A partir dessa análise preliminar, tomamos a decisão de eliminar da escala os itens 7 ("Participo ativamente em discussões de grupo") e 9 ("Faço perguntas quando não compreendo a matéria"). A decisão teve por base razões quer teóricas quer psicométricas. No que se refere a justificativas psicométricas, observou-se que o item 7 estava intercorrelacionado com o item 8 e revela uma precisão baixa $\left(R^{2}=0,15\right)$. Verificou-se também que o item 9 tinha o seu erro de mensuração intercorrelacionado com o de quatro outros itens e contribuía, assim, para o desajuste do modelo. Relativamente às justificativas teóricas, uma análise semântica dos dois itens revela estarem nitidamente associados às atividades de participação na aula (que são descritas na subescala PA). O item 8 ("Levanto o braço 


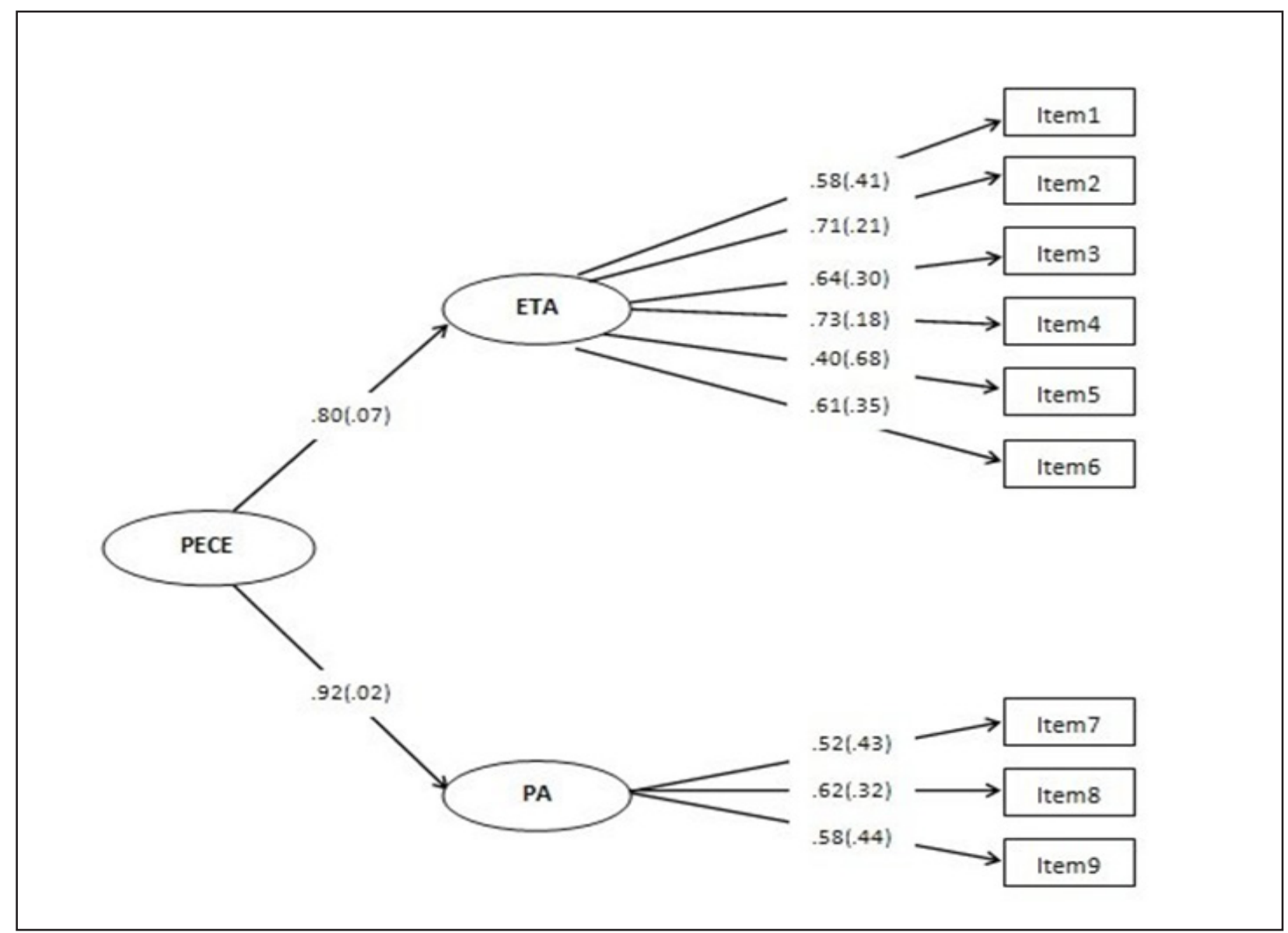

Figura 1. Carga fatorial estandardizada (variância do erro) na estrutura fatorial bidimensional.

para responder a uma pergunta") permaneceu nessa escala alternativa exploratória primeiro por não revelar problemas métricos. Embora o item encaixe na escala PA, o seu conteúdo semântico pode ser interpretado de uma forma mais genérica como envolvimento comportamental. Para além do argumento semântico, nota-se também que é um comportamento que faz parte do elenco tradicional de itens que medem envolvimento comportamental (e.g., Handelsman et al., 2005). Quer a subescala ETA de seis itens quer esta nova escala de sete itens revelam valores de alpha aceitáveis em termos de investigação (na ordem de 0,75 ).

\section{Discussão Geral}

A componente comportamental do envolvimento escolar traduz-se nas ações do aluno em contexto académico e nas suas práticas e atividades escolares (Veiga et al., 2012). Neste estudo, construiu-se e foi realizada a validação fatorial de uma escala que pretende avaliar as percepções dos alunos sobre o seu envolvimento comportamental escolar. A realização da AFE sugeriu a existência de uma estrutura bidimensional. AAFC confirmou essa estrutura. A percepção dos alunos sobre o seu envolvimento comportamental escolar assentou nos seus comportamentos de envolvimento no trabalho acadêmico, por um lado, e, por outro, nos seus comportamentos de participação na aula. A primeira dimensão é semelhante ao que é tradicionalmente tratado como envolvimento comportamental. A segunda dimensão que emergiu empiricamente pode representar algo novo, não previsto.

No entanto, os resultados obtidos no teste à adequabilidade do modelo hipotético permitiram somente aferir da sua plausibilidade para descrever os domínios em questão, não excluindo a existência de outras alternativas para a representação desses mesmos domínios (Browne \& Cudeck, 1993). Assim, a sua generalização é plausível para as variáveis consideradas, na forma como foram operacionalizadas. Finn (1989), por exemplo, defende a existência de diferenças qualitativas no tipo de envolvimento comportamental evidenciado pelos alunos, podendo o mesmo ir desde a resposta passiva às instruções do professor até ao envolvimento do aluno em atividades que exigem a sua iniciativa individual.

A dimensionalidade do construto de envolvimento tem-se focado nas dimensões comportamental, afetiva, cognitiva e, mais recentemente, agenciativa (Reeve \& Tseng, 2011; Veiga, 2013). O presente estudo parece representar, até agora, a primeira descrição de duas subdimensões comportamentais, mas será mesmo assim?

A dimensão agenciativa tem sido conceptualizada como o contributo construtivo dos alunos à instrução recebida, 
sendo simultaneamente relacionada à sua auto-percepção como um agente de ação (Reeve \& Tseng, 2011; Veiga, 2013). Níveis elevados de agência significam que um aluno demonstra iniciativa individual, intervém nas aulas, procura dialogar com os professores, quer fazendo perguntas quer oferecendo sugestões. Ou seja, a dimensão agenciativa parece constituir uma dimensão comportamental. No estudo de Veiga (2013), a dimensão comportamental centrou-se em comportamentos negativos, tais como interrupção intencional de aulas, comportamento inadequado para com os professores, faltar às aulas ou, ainda, demonstrar falta de atenção nas mesmas. Nesse sentido, a dimensão agenciativa pode estar a medir o que Skinner et al. (2009) referiram como envolvimento comportamental, enquanto o que Veiga tratou como envolvimento comportamental pode representar o que Skinner et al. chamaram de desinteresse. Futuros estudos de investigação deverão esclarecer a relação entre essas dimensões e clarificar a natureza da dimensão agenciativa como comportamental ou não.

Ao proceder-se a análises como as efetuadas no presente estudo, é possível observar modelos que, apesar de serem ajustados, originam subdimensões com níveis inaceitáveis de consistência interna. Como afirmou Michael Brannick (citado por Hurley et al., 1997), quando isso acontecer, estaremos perante uma situação análoga à da questão de significância estatística versus magnitude de efeito (em que se observam efeitos "significativos" embora triviais). Assim, a emergência da participação nas aulas como uma subdimensão do envolvimento pode representar um avanço para a investigação sobre envolvimento escolar, mas, como medida, a subdimensão deverá, em princípio, ser melhorada.

Uma maneira de melhorar a consistência interna de uma escala sumativa é através de um aumento no número de itens paralelos. A aplicação da fórmula geral de Spearman-Brown permite fazer uma estimativa da fidelidade das escalas se o número de itens for aumentado por um factor $N$ (Carmines \& Zeller, 1979). No caso atual, aplicando a fórmula $\left(r_{a b}=N r_{y y}\right.$ / $\left.\left[1+(N-1) r_{y y}\right]\right)$ e pressupondo um fator de aumento de 3 , chegamos a uma estimativa de consistência interna acima de 0,80 . Ou seja, se houver um total de nove itens (os três atuais mais seis itens paralelos), pode-se esperar observar um valor de consistência na ordem de 0,80 . Recomenda-se então um acréscimo no número de itens da escala PA antes do seu uso para fins de investigação.

Em virtude do baixo indicador de fiabilidade da escala PA, e até futuras investigações a melhorarem, procuramos efetuar análises adicionais para testar a viabilidade de uma escala unidimensional para a mensuração do envolvimento comportamental. A fím de testar essa possibilidade, recorremos à técnica de modelação de equações estruturais, em uma perspetiva exploratória. Os itens excluídos nessa solução eram componentes da subdimensão de participação na aula. Assim, até que haja uma melhoria da subdimensão de participação na aula, os investigadores poderão optar por empregar a subescala ETA de seis itens ou a escala de sete itens que emergiu na análise exploratória. As duas dimensões são semelhantes, quer em termos da consistência interna quer em termos dos conteúdos (Finn, 1993; Finn \& Rock, 1997; Fredricks et al., 2004; Johnson, Crosnoe, \& Elder, 2001; Jordan \& Nettles, 2000).
Futuros estudos deverão recorrer a outras técnicas que permitam analisar a validade convergente, divergente e preditiva das escalas apresentadas, dando seguimento a este que foi um primeiro passo na validação da escala de perceção dos alunos sobre o seu envolvimento comportamental escolar. Poderão também desenvolver as presentes escalas, no sentido de averiguar a existência de diferentes tipologias de comportamentos de envolvimento escolar, tais como a participação colaborativa, a participação autônoma ou a mera adesão às regras da sala de aula, no sentido de clarificar como se manifesta a intensidade e a qualidade do envolvimento dos alunos na vida escolar. Por outras palavras, conhecer melhor como o envolvimento escolar compromete, ou não, o projeto de vida futura dos alunos.

Futuras investigações poderão ainda procurar desvendar as diferenças nas percepções de alunos e professores sugeridas por Skinner et al. (2009). Se, como aludido, os professores desvalorizam sistematicamente o envolvimento dos alunos ou se, inversamente, os alunos sobrevalorizam o seu envolvimento, então estaremos perante implicações fortes para a necessidade de formação de professores e o planejamento da mesma.

\section{Referências}

Archambault, I., Janosz, M., Morizot, J., \& Pagani, L. (2009). Adolescent behavioral, affective, and cognitive engagement in school: Relationship to dropout. Journal of School Health, 79(9), 408 - 415. doi:10.1111/j.1746-1561.2009.00428.x

Bentler, P. M. (1990). Comparative fit indexes in structural models. Psychological Bulletin, 107(2), 238 - 246. doi. org/10.1037/0033-2909.107.2.238

Bentler, P. M., \& Bonnet, D. C. (1980). Significance tests and goodness of fit in the analysis of covariance structures. Psychological Bulletin, 88(3), 588 - 606.

Browne, M. W, \& Cudeck, R. (1993). Alternative ways of assessing model fit. In K. A. Bollen, \& J. S. Long (Eds.), Testing structural equation models (pp. 136 - 162). London: SAGE.

Carmines, E., \& Zeller, R. (1979). Reliability and validity assessment. Beverly Hills, CA: Sage.

Clark, L. A., \& Watson, D. (1995). Constructing validity: Basic issues in objective scale development. Psychological Assessment, 73, $309-319$.

Cortina, J. M. (1993). What is coefficient alpha? An examination of theory and applications. Journal of Applied Psychology, $78(1), 98-104$

Diniz, A. M., \& Almeida, L. S. (2005). Escala de Integração Social no Ensino Superior (EISES): Metodologia de construção e validação. Análise Psicológica, 4(23), 461 - 476.

Dotterer, A. M., \& Lowe, K. (2011). Classroom context, school engagement, and academic achievement in early adolescence. Journal of Youth and Adolescence, 40(12), 1649 - 1660. doi: 10.1007/s10964-011-9647-5

Finn, J. D. (1993). School engagement and students at risk. Washington, DC: National Center of Educational Statistics.

Finn, J. D., \& Rock, D. (1997). Academic success among students at risk for school failure. Journal of Applied Psychology, 82(2), $221-234$. 
Finn, J. D., Pannozzo, J. M., \& Voelkl, K. E. (1995). Disruptive and inattentive-withdrawn behavior and achievement among fourth graders. Elementary School Journal, 95, 421- 434.

Finn, J. D., \& Zimmer, K. S. (2012). Student engagement: What is it? Why does it matter? In S. L. Christenson, A. L. Reschly, \& C. Wylie (Eds.), Handbook of research on student engagement (pp. 97 -131). New York: Springer.

Fredricks, J., Blumenfeld, P., \& Paris, A. (2004). School engagement: Potential of the concept, state of the evidence. Review of Educational Research, 74(1), 59 - 109.

Handelsman, M., Briggs, W., Sullivan, N., \& Towler, A. (2005). A measure of college students' course engagement. Journal of Educational Research, 98, $184-191$.

Haynes, S., Richard, D., \& Kubany, E. (1995). Content validity in psychological assessment: A functional approach to concepts and methods. Psychological Assessment, 7(3), 238- 247.

Hirschfield, P. J., \& Gasper, J. (2011). The relationship between school engagement and delinquency in late childhood and early adolescence. Journal of Youth and Adolescence, 40(1), 3-22.

Hooper, D., Coughlan, J., \& Mullen, M. R. (2008). Structural equation modeling: Guidelines for determining model fit. Electronic Journal of Business Research Methods, 6(1), 53-60.

Hu, L. T., \& Bentler, P. M. (1999). Cutoff criteria for fit indexes in covariance structure analysis: Conventional criteria versus new alternatives. Structural Equation Modeling, 6(1), 1 - 55.

Hurley, A., Scandura, T., Schriesheim, C., Brannick, M., Seers, A., Vandenberg, R., \& Williams, L. (1997). Exploratory and confirrmatory factor analysis: Guidelines, issues, and alternatives. Journal of Organizational Behavior, 18, 667-683.

Johnson, M., Crosnoe, R., \& Elder, G. (2001). Students' attachment and academic engagement: The role of race and ethnicity. Sociology of Education, 74, 318 - 340. doi. org/10.2307/2673138

Jordan, W., \& Nettles, S. (2000). How students invest their time outside of school: Effects on school-related outcomes. Social Psychology of Education, 3, 217 - 243. doi: 10.1023/A:1009655611694
Li, Y., Zhang, W., Liu, J., Arbeit, M. R., Schwartz, S. J., Bowers, E. P., \& Lerner, R. M. (2011). The role of school engagement in preventing adolescent delinquency and substance use: A survival analysis. Journal of Adolescence, 34, 1181 - 1192. doi: 10.1016/j.adolescence.2011.07.00

Kindermann, T. A. (2007). Effects of naturally existing peer groups on changes in academic engagement in a cohort of sixth graders. Child Development, 78, 1186 - 1203.

Mulaik, S. A., James, L. R., van Alstine, J., Bennet, N., Lind, S., \& Stilwell, C. D. (1989). Evaluation of goodness-of-fit indices for structural equation models. Psychological Bulletin, 105(3), $430-445$.

Newmann, F. (1992). Student engagement and achievement in American secondary schools. New York: Teachers College Press.

Pilati, R., \& Laros, J. A. (2007). Modelos de equações estruturais em psicologia: Conceitos e aplicações. Psicologia: Teoria e Pesquisa, 23(2), $205-216$.

Reeve, J., \& Tseng, C. (2011). Agency as a fourth aspect of students' engagement during learning activities. Contemporary Educational Psychology, 36(4), 257-267. doi:10.1016/j. cedpsych.2011.05.002

Sijtsma, K. (2009). On the use, the misuse, and the very limited usefulness of Cronbach's alpha. Psychometrika, 74(1), $107-$ 120. doi: 10.1007/s11336-008-9101-0

Simons-Morton, B., \& Chen, R. (2009). Peer and parent influences on school engagement among early adolescents. Youth \& Society, 41(1), 3 - 25. doi: 10.1177/0044118X09334861

Skinner, E. A., \& Belmont, M. J. (1993). Motivation in the classroom: Reciprocal effects of teacher behavior and student engagement across the school year. Journal of Educational Psychology, 85(4), 571 - 581.

Skinner, E. A., Kindermann, T., \& Furrer, C. (2009). A motivational perspective on engagement and disaffection: Conceptualization and assessment of children's behavioral and emotional participation in academic activities in the classroom. Educational and Psychological Measurement, $69(3), 493-525$.

Steiger, J. H. (1990). Structural model evaluation and modification. Multivariate Behavioral Research, 25(2), 173 - 180.

Tavares, D. (2007). Escola e identidade profissional: O caso dos técnicos de cardiopneumologia, Lisboa: Colibri / IPL.

Veiga, F. H. (2013). Envolvimento dos alunos na escola: Elaboração de uma nova escala de avaliação. International Journal of Developmental and Educational Psychology, 1(1), 441-450.

Veiga, F., Galvão, D., Festas, I., \& Taveira, C. (2012). Envolvimento dos alunos na escola: Variáveis contextuais e pessoais: Uma revisão de literatura. Psicologia, Educação e Cultura, 16(2), $36-50$.

Wenger, E. (2007). Communities of practice: Learning, meaning and identity. Cambridge: Cambridge University Press.

Recebido em 07.11.2014

Primeira decisão editorial em 16.06.2015

Versão final em 15.07.2015

Aceito em 14.09.2015 